\title{
Turbulent jet through porous obstructions under Coriolis effect: an experimental investigation
}

\author{
Francesca De Serio ${ }^{1,2}\left(\mathbb{0} \cdot\right.$ Roni H. Goldshmid ${ }^{3}$. Dan Liberzon ${ }^{3} \cdot$ Michele Mossa $^{1,2} \cdot$ M. Eletta Negretti $^{4}$. \\ Giuseppe R. Pisaturo ${ }^{5}$. Maurizio Righetti ${ }^{5}$. Joel Sommeria ${ }^{4}$. Donatella Termini ${ }^{6} \cdot$ Thomas Valran $^{4} \cdot$ Samuel Viboud $^{4}$
}

Received: 11 May 2021 / Revised: 11 August 2021 / Accepted: 31 August 2021 / Published online: 5 October 2021

(c) The Author(s) 2021

\begin{abstract}
The present study has the main purpose to experimentally investigate a turbulent momentum jet issued in a basin affected by rotation and in presence of porous obstructions. The experiments were carried out at the Coriolis Platform at LEGI Grenoble (FR). A large and unique set of velocity data was obtained by means of a Particle Image Velocimetry measurement technique while varying the rotation rate of the tank and the density of the canopy. The main differences in jet behavior in various flow configurations were assessed in terms of mean flow, turbulent kinetic energy and jet spreading. The jet trajectory was also detected. The results prove that obstructions with increasing density and increased rotation rates induce a more rapid abatement of both jet velocity and turbulent kinetic energy. The jet trajectories can be scaled by a characteristic length, which is found to be a function of the jet initial momentum, the rotation rate, and the drag exerted by the obstacles. An empirical expression for the latter is also proposed and validated.
\end{abstract}

\section{Graphic abstract}

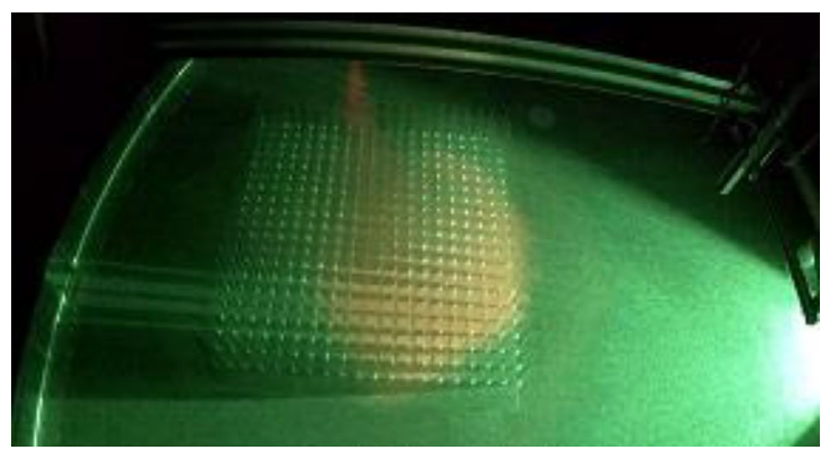

Francesca De Serio

francesca.deserio@poliba.it

1 DICATECh, Polytechnic University of Bari, Bari, Italy

2 CoNISMa, Rome, Italy

3 Faculty of Civil and Environmental Engineering, The Technion, Haifa, Israel

4 CNRS, Grenoble INP, LEGI, Univ. Grenoble Alpes, Grenoble, France

5 Faculty of Science and Technology, Free University of Bozen, Bolzano, Italy

6 Department of Engineering, University of Palermo, Palermo, Italy

\section{Introduction}

Rivers and wastewater discharge into lakes and coastal waters often form turbulent jets. Long-shore and cross-shore coastal currents can also be intended as jet-like flows in some cases when constrained by local topography or manmade structures. These types of environmental flows all have a profound effect on aquatic ecosystems due to the transport of turbulence, tracers, and sediment particles. At the same time, the surrounding environment affects their mixing and dispersion processes. Our ability to predict their behavior strongly depends on our capacity to describe how jets interact with the real environment they discharge into. 
For this reason, the action played by Coriolis force cannot be disregarded. Similarly, the presence of obstructions in the receiving water body must also be given due consideration. Examining these mutual interactions could certainly be of help in mitigating undesired impacts and in developing best environmental management practices.

Mixing processes are rather well understood for unobstructed jets and are mainly controlled by the initial jet characteristics, the boundary conditions, and the hydrodynamic features of the ambient current (Smith and Mungal 1998; Nepf 2012; Liberzon and Fernando 2014). When such jets are discharged in the presence of rotation, the Coriolis force changes the orientation of turbulent eddies, especially affecting the energy cascading process (Lin and Atkinson 2000; Moisy et al. 2011). For density-driven jets, mixing and entrainment depends on bottom slope, Froude and Reynolds number (Fernando and Ching 1993; Praud et al. 2006; Thomas and Linden 2007; Cossu et al. 2010).

Our knowledge of the effect of vegetation on flows, in general, is rather extensive. The vegetation canopy, characterized particularly by its density and geometry (Oldham and Sturman 2001; Nepf et al. 1997; Ghisalberti and Nepf 2005; Poggi et al. 2009; Nepf 2012; Termini 2015, 2018), interacts with the mean and turbulent flow, perturbing both advection and dispersion (Raupach and Thom 1981; Ben Meftah et al. 2015; De Serio et al. 2018; Righetti 2008). For canopy flows, the formation of coherent structures has been studied also numerically, e.g. Bailey and Stoll (2016).

Momentum jets obstructed by vegetation have been investigated only recently (Ben Meftah et al. 2015; Ben Meftah and Mossa 2016). It is well known that, when released in an unobstructed flow, the momentum jet experiences entrainment, while detrainment is observed only for density jets. Results by Mossa and De Serio (2016) and Mossa et al. (2017) demonstrated that the vegetation canopy is capable of inducing detrainment for strong momentum jets, which is unexpected behavior.

Most previous experimental studies of both obstructed jets in absence of rotation and unobstructed jets in rotating background have been conducted mainly in small-scale facilities (Etling and Fernando 1994; Lin and Atkinson 2000; Nepf 2012; Termini 2015). Even when large-scale facilities have been used the experiments have never investigated the joint influence of jets, obstacles, and rotation (Moisy et al. 2011; Ben Meftah et al. 2015; Davarpanah et al. 2020).

Although there is a good understanding of how turbulent jets interact either with rotation alone or with vegetation alone, to the best of our knowledge no one has so far experimentally investigated jets that are affected simultaneously by vegetation and the Coriolis force, always present in the field. Hence, this is the main aim and the novel aspect of the present paper. Although in real sea, due to real scales, the direct effect of rotation on turbulence induced by an obstructed pattern is expected quite negligible, the mean flow is modified by rotation and consequently, also the transport and spreading of turbulent kinetic energy and scalars (tracers) by the mean flow are modified accordingly.

As known, geophysical flows are generally characterized by time scales $\tau$ such that $\tau \geq 1 / \omega$, where $\omega$ is the Earth's rotation rate. Being geophysical flows often shallow, with the horizontal length scale $L$ much larger than the vertical one $H$, for the horizontal and vertical velocities we have $U>>W$, where $U$ and $W$ are the horizontal and vertical velocity scales, respectively. Consequently, such flows are usually considered bi-dimensional, developing in the horizontal plane. They are characterized by a Rossby number $R o=U /(2 \omega L)$ which is less than unity, indicating that the Coriolis effect, i.e. rotation, prevails on the advective motion, and thus can effectively modify the flow dynamics.

To evaluate some effects induced by the Coriolis force on a turbulent flow, a turbulent Rossby number can be defined analogously as $R o^{\prime}=u_{t}^{\prime} /(2 \omega l)$, being $u_{t}^{\prime}$ a turbulent velocity scale associated with an integral length scale $l$. Considering the typical order of magnitude for $u_{t}^{\prime}$ and $\omega$, we generally get $R o^{\prime}>>1$. Signifying the turbulent flow is unperturbed by Earth rotation (Godeferd and Moisy 2015), as the rotation timescale of $1 / \omega>>l / u_{t}$ ' which is the timescale over which the turbulent structure evolves.

Addressing mesoscale flows, such as coastal flows, with typical horizontal length scales of $\mathrm{O}(10-100) \mathrm{km}$ and velocity scales of $\mathrm{O}(0.1-1) \mathrm{m} / \mathrm{s}$, the Rossby number tends to approach unity $R o \approx 1$. In this case, the inertial and the rotation time scales become comparable so that a mutual influence between the mean flow and the rotation may become important. When such flows are in presence of obstructions, the turbulent velocity scale has an order of magnitude $u_{t}{ }^{\prime}=(0.01 \div 0.1) U$ (De Serio et al. 2018). As for the integral length scale $l$, it can vary significantly (Nepf 2012; De Serio et al. 2018), depending on the density and location of the obstructions, spanning over $\mathrm{O}(0.1-100) \mathrm{m}$. Consequently, also $R o$ ' can vary greatly. In such cases, when $R o$ ' exceeds unity, the turbulent structures are unaffected by rotation directly. Instead, it is the transport and spreading by the mean flow of the turbulent fluctuations that are expected to be modified by rotation. Based on these scales, the examined conditions can be considered representative of rivers or wastewater discharges flowing into coastal waters through reeds and marsh beds, as well as offshore wind plants located in estuarine areas.

The paper outline is as follows. The experimental apparatus, the executed tests, and the methods used for processing experimental data are presented in Sect. 2. Section 3 includes examination and discussion of the obtained results in terms of the mean flow, jet width, turbulent kinetic energy, and transport of turbulent kinetic energy by the mean flow. Finally, some considerations about the jet trajectory are provided in Sect. 4. 


\section{Experimental setup and measurement technique}

The experiments, carried out in the large-scale Coriolis rotating Platform, were performed using two configurations: in the first, the jet was only affected by background rotation; in the second, the jet flows through a canopy field consisting of rigid rods, mimicking rigid and emergent vegetation. To allow a comparison, the unobstructed and obstructed jets were examined under numerous identical clockwise rotation velocities of the tank $\Omega=2 \pi / T, T$ being the rotation period.
Besides the rotation rate, an additional variation parameter was the spacing between the rods, $s$.

The Coriolis Platform has a diameter of $D=13 \mathrm{~m}$, the working water depth was set at $H=0.80 \mathrm{~m}$. The momentum jet was released from a dedicated pipe, rigidly fixed at $0.40 \mathrm{~m}$ depth (Fig. 1), fed from a constant head tank. The outlet ensured a horizontal release of the jet with an initial diameter of $d_{j}=0.08 \mathrm{~m}$ (internal pipe diameter) and initial flowrate $Q_{j}$. A specially designed canopy was placed in the tank for the obstructed flow configuration. The rigid, emergent vegetation stems were modeled by an array of plastic transparent rods
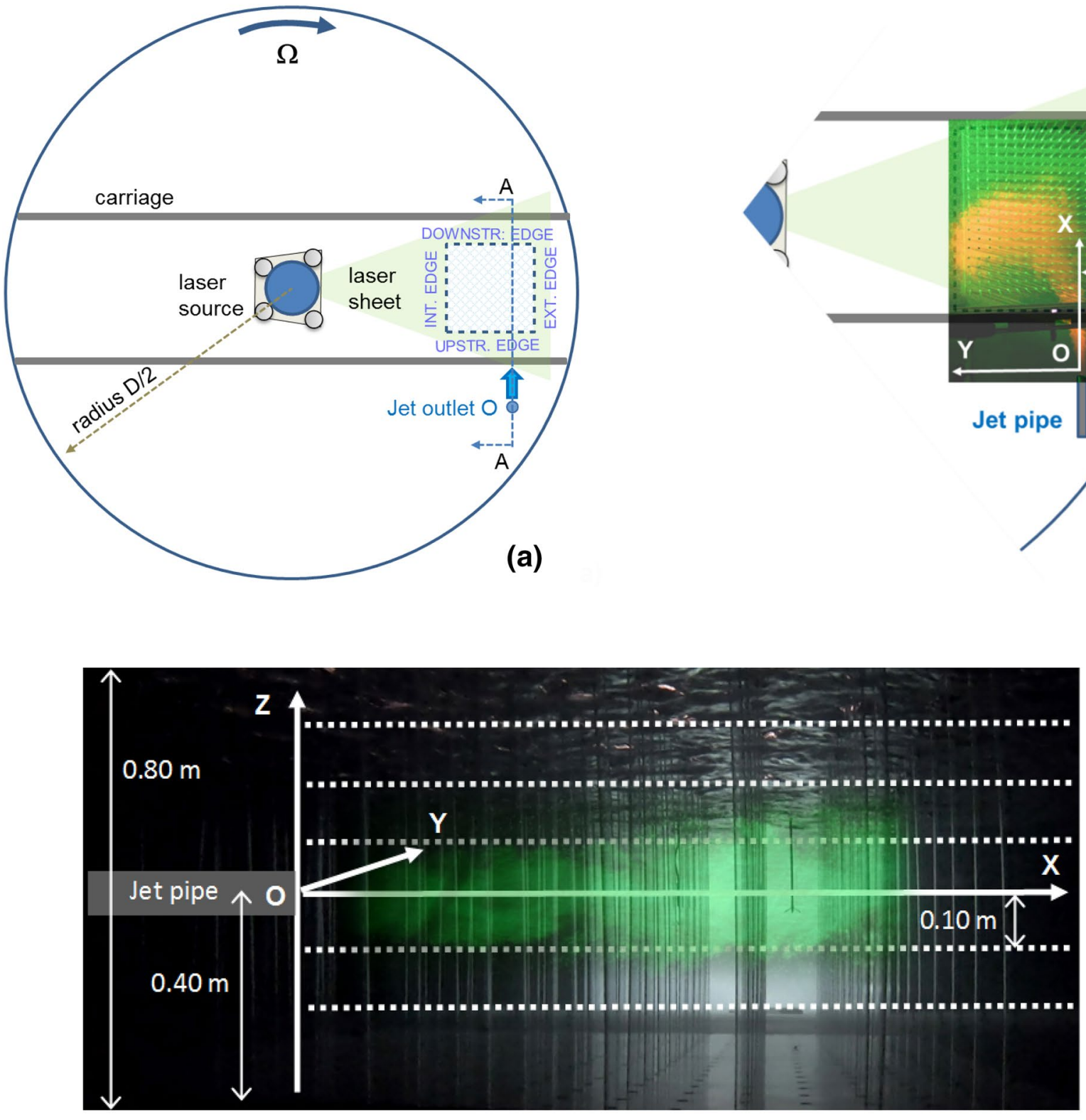

(c)

Fig. 1 Experimental setup: a Schematic view of the LEGI tank with location of jet outlet and panel; b Detail of the obstructed area and example of the issued jet, here dyed for visualization; c Prospective view of the vertical plane AA with location of the jet outlet and dotted lines corresponding to all the investigated horizontal layers (jet spreading in green) 
(made of Plexiglass $®$ ) $d=0.02 \mathrm{~m}$ in diameter, arranged on a $2 \mathrm{~m} \times 2 \mathrm{~m}$ panel fixed at the bottom of the tank. The rods were manually mounted on the panel inside pre-drilled holes and constituted a regular pattern with the center-to-center distance $s$. The panel was placed in the tank as shown in Fig. 1. The center of the jet outlet (O) was $1 \mathrm{~m}$ away from the upstream edge of the panel and $0.77 \mathrm{~m}$ from its external edge.

The instantaneous measurements of the velocity field were made using a Particle Image Velocimetry (PIV) system. The laser source of the PIV (continuous Nd Yag laser, $532 \mathrm{~nm}, 25 \mathrm{~W}$ ) was mounted in the center of the tank on a vertical axis. The laser sheet was set in a horizontal plane and was $5 \mathrm{~mm}$ thick. It covered a surface of $3 \times 3 \mathrm{~m}^{2}$ by means of an optical lens of $60^{\circ}$ angle (Fig. 1a,b). Polyamide particles (Orgasol®) with density of $1016 \mathrm{~kg} / \mathrm{m}^{3}$ and diameter of $60 \mu \mathrm{m}$ were used to seed the flow. They were added and mixed to the water in the head tank feeding the jet. The images were acquired using three synchronized CMOS cameras (two PCO ${ }^{\circledR}$ filming at $2560 \times 2160$ pixels resolution and one Falcon ${ }^{\circledR}$ filmings at $2432 \times 1728$ pixels resolution) mounted above the water surface, fixed on rigid supports located at $4.29 \mathrm{~m}, 4.74 \mathrm{~m}$, and $4.87 \mathrm{~m}$ from the bottom, correspondingly. They filmed at a frequency of $33 \mathrm{~Hz}$ for $60 \mathrm{~s}$, resulting in a total of 2000 images per measurement. This PIV acquisition frequency was properly selected to avoid possible disturbances of the free surface deformations on the measurements, that is the time delay between two successive PIV images was set to be much smaller than the typical time scale of the free surface disturbances induced by the jet.

The position of the cameras ensured the three fields of view (FOV1 corresponding to PCO1, FOV2 corresponding to $\mathrm{PCO} 2$, and FOV3 corresponding to FALCON) partially overlapped, to ensure full coverage of the jet propagation path (Fig. 2). The reference system has origin at the jet outlet $\mathrm{O}$, with the $X$ and $Y$ axes in the horizontal plane, $X$ directed along the jet longitudinal axis, and $Y$ in the transverse direction, oriented towards the center of the tank; the $Z$-axis is vertical and positive upward (Fig. 1c). To map the velocity fields in the examined volume of water, the $2 \mathrm{D}$ velocity fields were acquired at six equispaced horizontal layers (Fig. 1c). Nevertheless, for the scope of the present study, only data assessed in layer $Z=0 \mathrm{~m}$, i.e. at mid-depth, are examined.

The control number characterizing our experiments is the jet-based Rossby number $\operatorname{Ro}_{j}=u_{j} /(2 \Omega D)$, where $u_{j}$ is the jet velocity at the outlet (along $X$ ). We also define a jet Reynolds number $\operatorname{Re}_{j}=u_{j} d_{j} / \nu$, with $\nu$ being the water kinematic viscosity. In the analyzed experiment, the jet flow rate $Q_{j}=0.006 \mathrm{~m}^{3} / \mathrm{s}$ was used, corresponding to $u_{j}=1.144 \mathrm{~m} / \mathrm{s}$ setting the jet Reynolds number is in all cases at $\operatorname{Re}_{j}=91514$, indicating a fully turbulent jet flow. Experiments executed at two different rotation rates are considered in this paper, $\Omega=0.070 \mathrm{rad} / \mathrm{s}$ and $\Omega=0.105 \mathrm{rad} / \mathrm{s}$, respectively corresponding to $T=90 \mathrm{~s}$ and $T=60 \mathrm{~s}$. For each value of $T$, two different

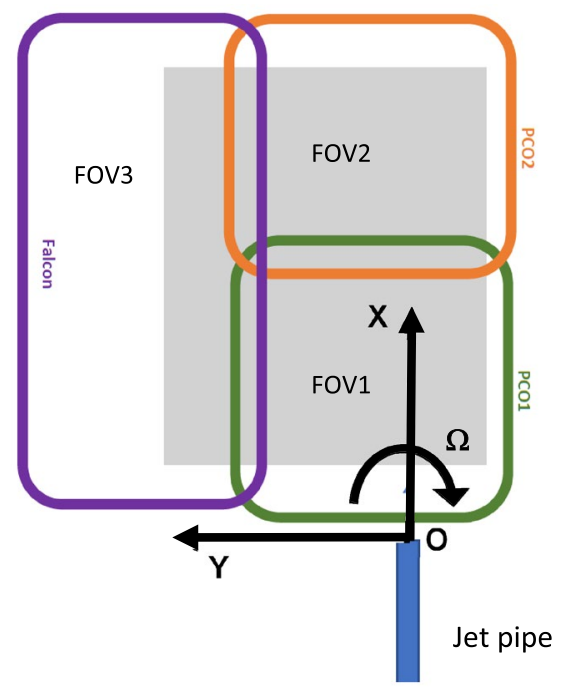

Fig. 2 Top view of cameras FOVs, grey rectangle marks the location of the canopy

Table 1 Executed tests with fundamental parameters

\begin{tabular}{llll}
\hline EXP & $\mathrm{T}[\mathrm{s}]$ & $\mathrm{Ro}_{\mathrm{j}}$ & Obstruction spacing s [m] \\
\hline EXP 15 & 90 & 0.63 & No obstructions \\
EXP 19 & 90 & 0.63 & 0.20 (low rods density) \\
EXP 23 & 90 & 0.63 & 0.10 (high rods density) \\
EXP 16 & 60 & 0.42 & No obstructions \\
EXP 18 & 60 & 0.42 & 0.20 (low rods density) \\
EXP 24 & 60 & 0.42 & 0.10 (high rods density) \\
\hline
\end{tabular}

The experiments are listed with their original names, as they appear in the ZENODO repository

densities of the obstructed array were examined. The low rods density was $n=25 / \mathrm{m}^{2}$ for rods distanced $s=0.20 \mathrm{~m}$ from each other, resulting in a solid volume fraction occupied by obstacles of $\phi=0.79 \%$. The high rods density was $n=100 / \mathrm{m}^{2}$ for rods distanced $s=0.10 \mathrm{~m}$, resulting in solid volume fraction of $\phi=3.14 \%$ (Nepf 2012). Such values of $\phi$ in the range $[0.001 \div 0.04]$ can be considered representative of reeds and marsh beds (Liu et al. 2021) in a coastal environment.

Table 1 lists all the analyzed experiments (EXP), for which similar analysis procedure was implemented. The jet-based Rossby numbers are both of order of magnitude $\mathrm{O}\left(10^{-1}\right)$, allowing us to evaluate the effect of rotation on the jet mean flow, expected to be more pronounced in the experiments characterized by $T=60 \mathrm{~s}$.

For the sake of brevity, experiments without rotation carried out as control tests, are not described in the present study, as they showed typical and expected trends (Ben Meftah et al. 2015; Mossa and De Serio 2016; Mossa et al. 2017). Please refer to De Serio et al. (2019) for details. The adopted 
modus operandi for processing the PIV data from all examined experiments was as follows. The PIV images acquired by each camera were successively coupled and correlated, to obtain the PIV measured velocity fields in the horizontal plane. As for the PIV images, velocities along the $X$ direction are named $u$, while those in the $Y$ direction are named $v$. The open-source ParaPIV software (Wang 2018) running in Matlab® was used to process the acquired images. Extensive parallelization (16 cores) was implemented to reduce computational times. The interrogation window size was set at $32 X 32$ pixels with a step size of 16 pixels. Two passes were used. The first pass was 64 X64 pixels with a step size of 32 , and the second pass was $32 \times 32$ pixels with an overlap of 16 pixels. The uniform distribution of the canopy base panel was used as the real-world-coordinates translation grid. The equidistant holes (in the bottom panel) were exposed completely in EXP15 and their centers were detected. The centers were used to perform the geometric transformation of all three cameras' images, aligning them on the same grid and correcting minor optical distortions. The same spatial optical alignment calibration parameters were used for all the experiments. For reference, the average PIV calibration parameters were: PCO1 $15.4\left[\mathrm{px} \mathrm{cm}^{-1}\right], \mathrm{PCO} 213.6\left[\mathrm{px} \mathrm{cm}^{-1}\right]$, and Falcon $8.41\left[\mathrm{px} \mathrm{cm}^{-1}\right]$. The resulting spatial resolution of the velocity vectors was $1 \mathrm{~cm}$ and the velocity accuracy was $1 \%$.

\section{Results and discussion}

Reaching a steady flow condition of the jet was verified first, before implementing time-averaged analysis of the assessed data. Figure 3 shows, as an example, the cumulative average and cumulative $r m s$ of $u$ velocity component in one selected point of the jet core, respectively for EXP15, EXP19, and EXP23. It is quite evident that an asymptotic value is achieved in all cases.

\subsection{Jet velocity, width, and turbulent kinetic energy}

A local reference system is conveniently placed to better follow the jet behavior, that is a curvilinear abscissa $X s$ along the jet trajectory with its origin at the jet outlet. The jet behavior was studied in the zone of established flow (Sami et al. 1967) for all the experiments, starting at $X s=X s_{0}$. The value of $X s_{0}$ is detected as the first distance from the outlet, greater than $10 d_{j}$ (Sami et al. 1967), where the transversal profile of the streamwise velocity approached the typical Gaussian shape. Specifically, the values of $X s_{0}$ are $X s_{0}=1.19 \mathrm{~m}, 1.15 \mathrm{~m}, 1.95 \mathrm{~m}, 1.42 \mathrm{~m}, 1.68 \mathrm{~m}$, and $1.66 \mathrm{~m}$, respectively for experiments EXP15, EXP16, EXP19, EXP18, EXP23, EXP24. Different definitions are generally used to identify the jet trajectory, and as of today, none is considered as the standard (New et al. 2006). Some researchers (e.g. Ben Meftah et al. 2015) define the jet trajectory as the locus of maximum jet velocity, while others (New et al. 2006) as the streamline starting from the center of the jet. In the present work, the trajectory is identified as the streamline starting from the central point of the crosssection at $X s=X s_{0}$ (Mossa et al. 2021). In the local reference system, at each point of the curvilinear abscissa $X s$, the radial axis $Y s$ is also placed (positive towards the center of rotation). $U s$ and $V s$ are the streamwise and spanwise velocity components along $X s$ and $Y s$, respectively. The timeaveraged velocity fields and the detected jet trajectories are displayed in Fig. 4. Specifically, for all the experiments investigated (Table 1), some selected transversal profiles are plotted in the left panels of Fig. 4, while the overall velocity fields together with the jet trajectory are plotted in the right panels of Fig. 4. As previously written, the possible misalignment of the cylinders along the edges of the FOVs, essentially due to the different camera perspectives, was adequately taken into account in the data processing. The selected cross-sections are equidistant, at different $X s_{i}$, named with increasing values of $-i$ suffix. In all cases, it is evident that the rotation affects the jet deflecting its trajectory. As expected, the jet velocity reduces with increasing distance from the outlet. We observe the maxima values of the velocity generally along the jet trajectory, especially in the fields of view FOV1 and FOV2. In the portion of the jet covered by FOV3, especially for EXP15, EXP16, and EXP19, the maxima velocities are slightly shifted towards the center of rotation.

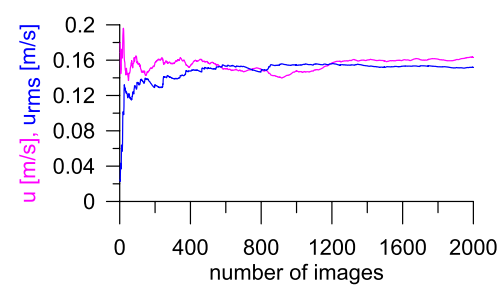

(a)

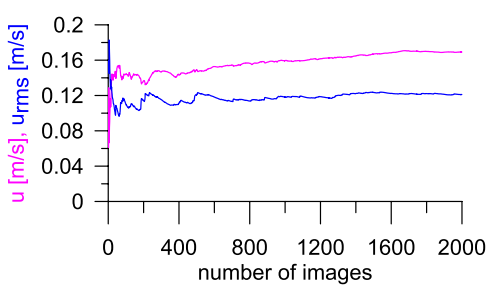

(b)

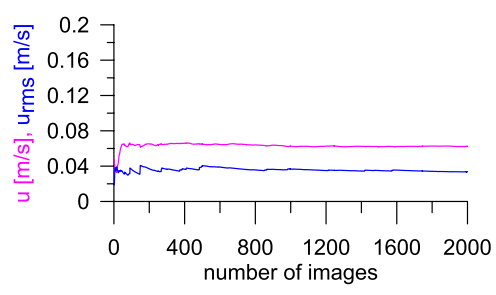

(c)

Fig. 3 Cumulative averages of $u$ and of $u_{r m s}$ for the points (a) $X=2.18 \mathrm{~m}$ and $Y=0.41 \mathrm{~m}$ of EXP15, (b) $X=1.93 \mathrm{~m}$ and $Y=0.43 \mathrm{~m}$ of EXP19 and (c) $X=1.96 \mathrm{~m}$ and $Y=0.46 \mathrm{~m}$ of EXP23 

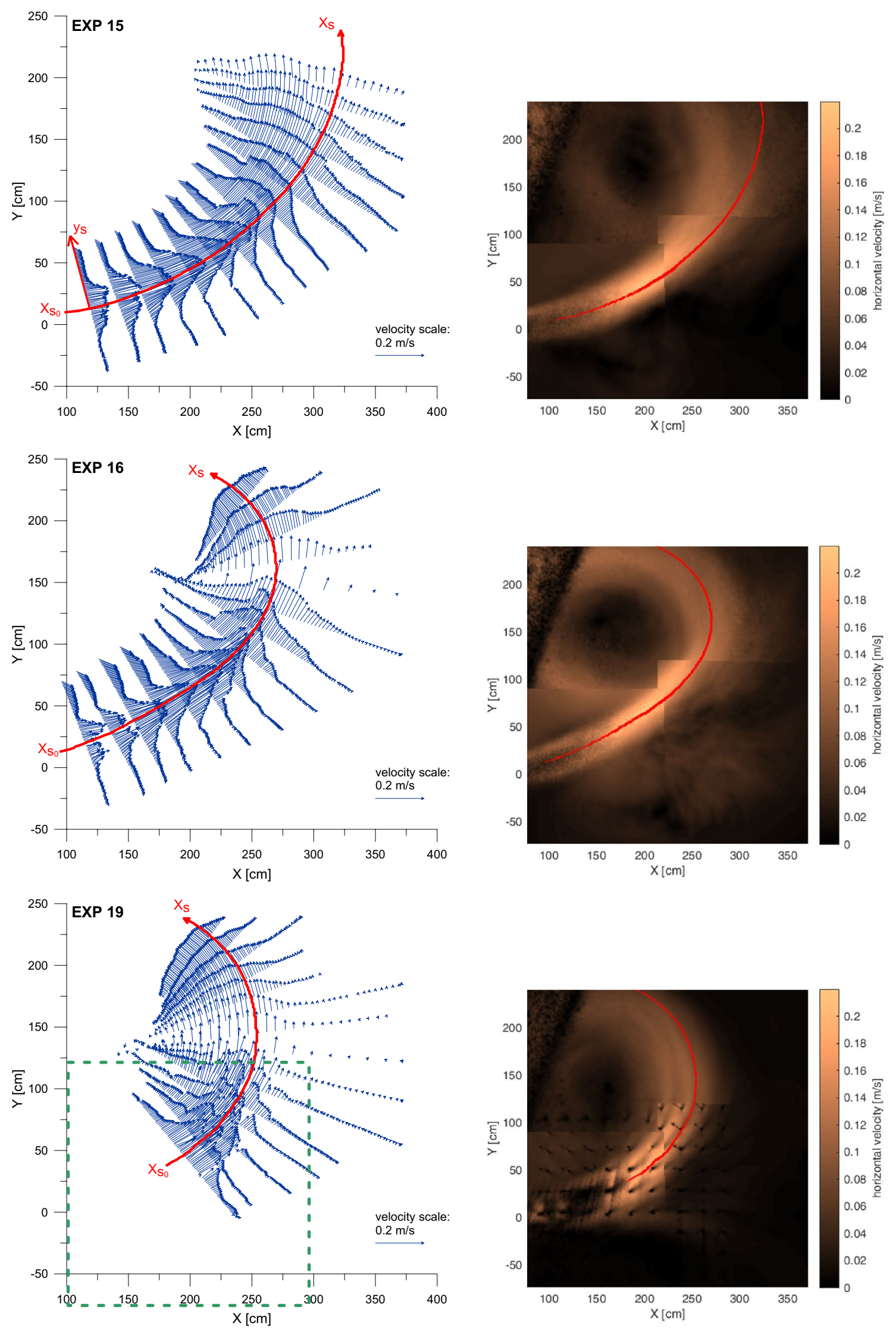

Fig. 4 Plots of selected transversal profiles of the time-averaged velocity at varying $X s$ abscissa (left) and of the overall velocity field with location of jet trajectory (right). Green dotted shape delimits the obstructed panel 

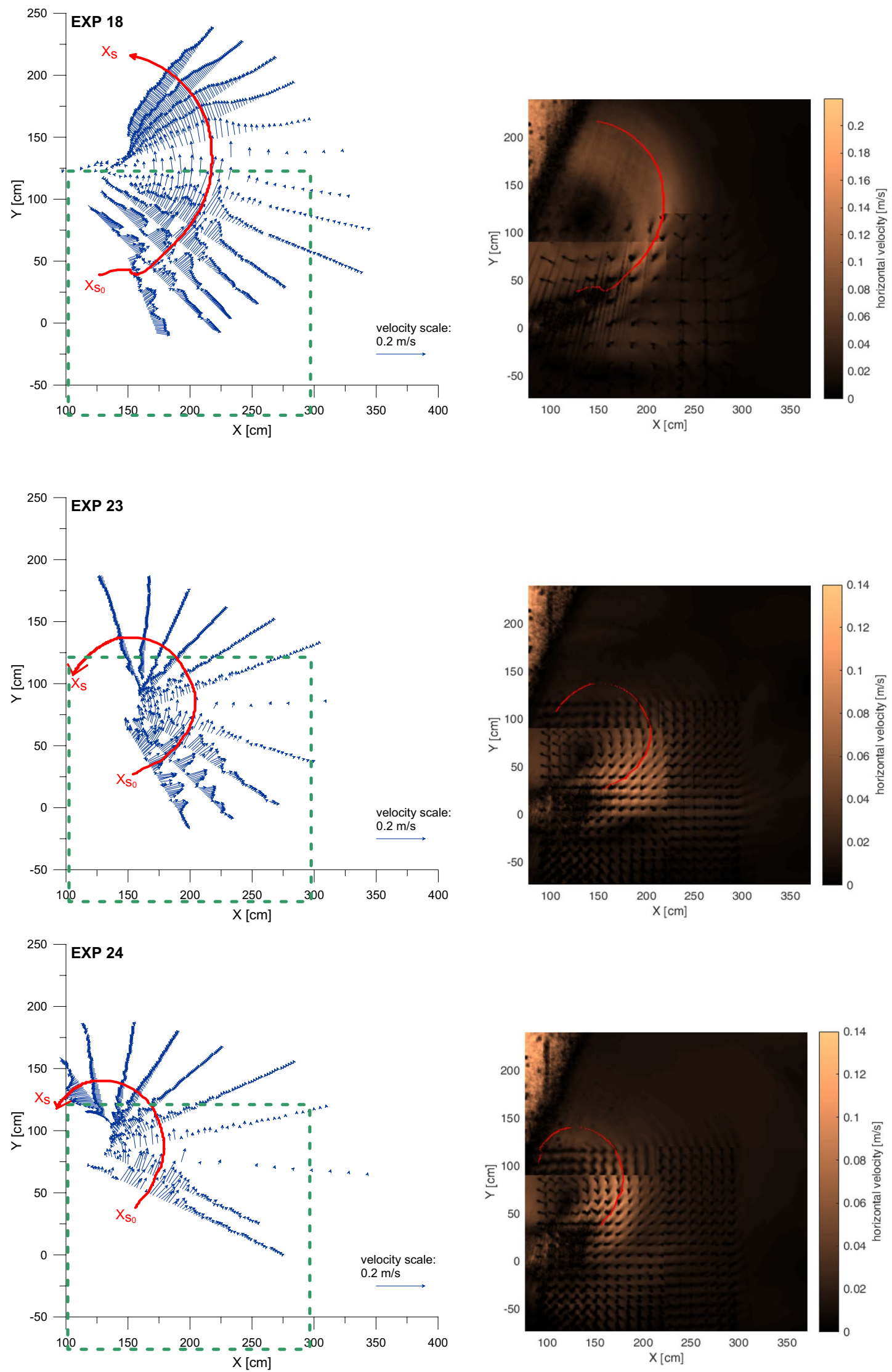

Fig. 4 (continued) 
Compared with the unobstructed cases, the presence of rods array determines a more rapid decrease of the velocity magnitude along the jet trajectory. The rate of velocity decrease is higher when the array density is higher (EXP23 and EXP24), as expected due to the increased blocking effect of the rods and the reduced drag effect. The comparison between experiments with identical rods array density (such as EXP19 and EXP18) shows higher velocity reduction rate at faster rotation rates, thus manifesting jet inertia limitation by Coriolis effect.

In Fig. 5 we plot the time-averaged streamwise velocities normalized by their maxima values, i.e. $U s / U s_{\max }$, varying along $Y s$ (rated by $X s-X s_{0}$ ). Figure 5 shows steep gradients near the jet edges in all the experiments. For EXP15 and EXP16, the curves $U s / U s_{\max }$ tend to collapse on a single curve as expected for free jets (Hussein et al. 1994) with a peak close to the center of the jet, thus following the jet self-similarity behavior. The Gaussian shape of the curve typical for free jets is also observed in the obstructed experiments for sections not intercepting the canopy (magenta marked trends in Fig. 5). On the contrary, when a section intercepts the canopy, $U s / U s_{\max }$ has an undulating trend, with lower values corresponding to the location of obstructions and higher values to the free space between two consecutive obstructions (De Serio et al. 2018). Such undulating behavior is clearly observed in EXP19 and EXP18 for sections $X s_{1} \div X s_{7}$, as well as in EXP23 and EXP24 especially for sections $X s_{1} \div X s_{5}$ (green marked trends in Fig. 5). Nevertheless, the envelope curve resembles the Gaussian shape. The symmetry with respect to the jet axis is generally preserved in EXP15 and EXP16. In presence of obstructions, the jet symmetry is almost lost inside the canopy. Moreover, in the obstructed cases the jet has the tendency to spread more towards the center of rotation, as revealed by the position of the Gaussian toe; such shift also intensifies with the increasing obstruction density.

Referring to the spanwise velocities $V s$, they are one order of magnitude lower than the streamwise ones for all cases. In Fig. 6 they are plotted along each examined profile $Y s /\left(10 d_{j}\right)$ as dimensionless $V s / 0.1 u_{j}$. Figure 6 demonstrates that the spanwise velocity components are mainly antisymmetric with respect to the jet axis, supporting previous observations by Mossa and De Serio (2016). A more detailed examination of the profiles gives information on entrainment or detrainment processes. In the experiments without obstructions (EXP15 and EXP16), for sections $X s_{1} \div X s_{9}$, the spanwise velocities in
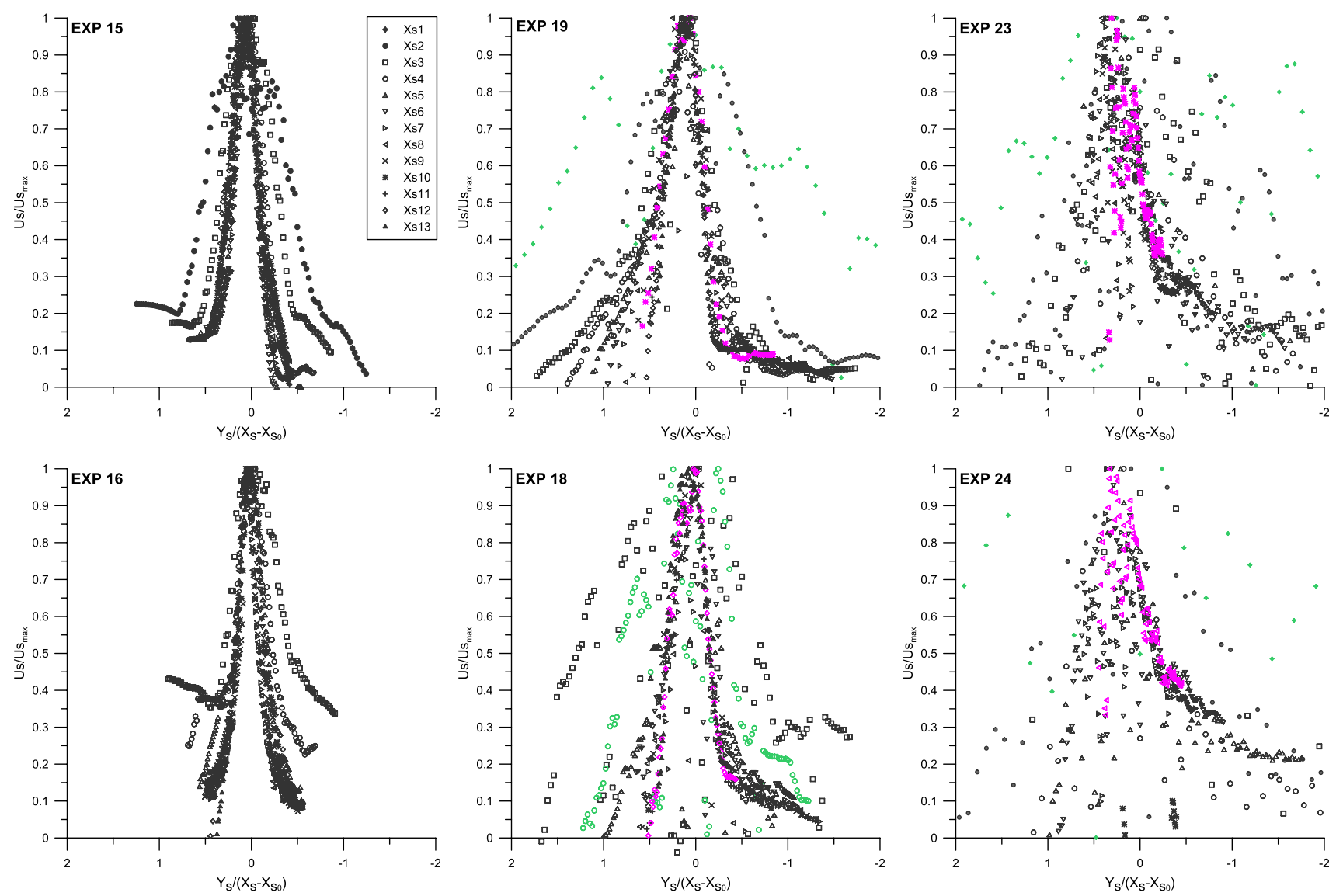

Fig. 5 Profiles along the examined sections of the normalized streamwise jet velocities. Top row $T=90 \mathrm{~s}$ and bottom row $T=60 \mathrm{~s}$. Colored plots in the online version of the paper 
the core region are always directed towards the center of the jet, with similar entrainment as in free jets. Outside the core region, mainly for external points at $Y s /\left(10 d_{j}\right)<-0.25, V s$ values are negative, i.e. directed outwards. For reference, see the trends marked in orange in Fig. 6 for EXP15 and EXP16. Starting from section $X s_{9}$ and up to section $X s_{13}$, the jet trajectory represents a specific reference line. In fact, especially for EXP15, internal points $\left(Y_{s} /\left(10 d_{j}\right)>0\right)$ are characterized by positive $V s$ values, while in both EXP15 and EXP16 external points $\left(Y s /\left(10 d_{j}\right)<0\right)$ are characterized by negative $V s$ (azure marked trends in Fig. 6). These observations show a tendency of the jet to a transversal spreading, mainly towards the external edge, suggesting that rotation also induces a detrainment at a distance from the outlet where the Coriolis effect prevails over the inertial one. Such distance is investigated in more detail in Sect. 3.2. In the obstructed cases (EXP19 and EXP18), the $V s$ values are mainly positive for $Y s /\left(10 d_{j}\right)>0$, with exception for the initial sections of the set, and are generally negative for $Y s /\left(10 d_{j}\right)<0$, thus showing a substantial detrainment towards the ambient flow. Apart from the last sections of the set, where the jet tends to close on itself, an analogous detrainment is also observed for the velocities in the obstructed cases EXP23 and EXP24 (see orange marked trends in Fig. 6 for EXP19, EXP18, EXP23, and EXP24). Such behavior agrees well with observations of Mossa and De Serio (2016), where jets interacting with rigid obstructions in absence of rotation were characterized by detrainment.

In each investigated cross-section, the jet transversal width $B$ was obtained, defined as the total distance from the two points along $Y s$ where the streamwise velocity tends to become negligible. We assume such condition is reached when $U s=U s_{m a x} / e, e$ being the Euler number. The obtained values of $B$ are plotted in Fig. 7 along $X s / d_{j}$, showing a linear trend with increasing distance $X s$. The linear relation corresponds to typical free jets released in quiescent fluid, where the coefficient of proportionality between the jet radius and the downstream distance from the outlet is 0.1 .

For same rotation rate $T=90 \mathrm{~s}$, the jet widths are comparable for the cases EXP15 and EXP19 along the same distances $X s / d_{j}=25 \div 40$, rather for EXP23 the jet starts dispersing closer to the outlet. Moreover, for equal distances, the jet width is greater in EXP23 than in EXP19, consistently with the higher density of the rods, a condition for which the jet exhibits larger transversal deviation. Aanalogous behavior is observed also for the corresponding experiments with $T=60 \mathrm{~s}$. $B$ values are comparable for same
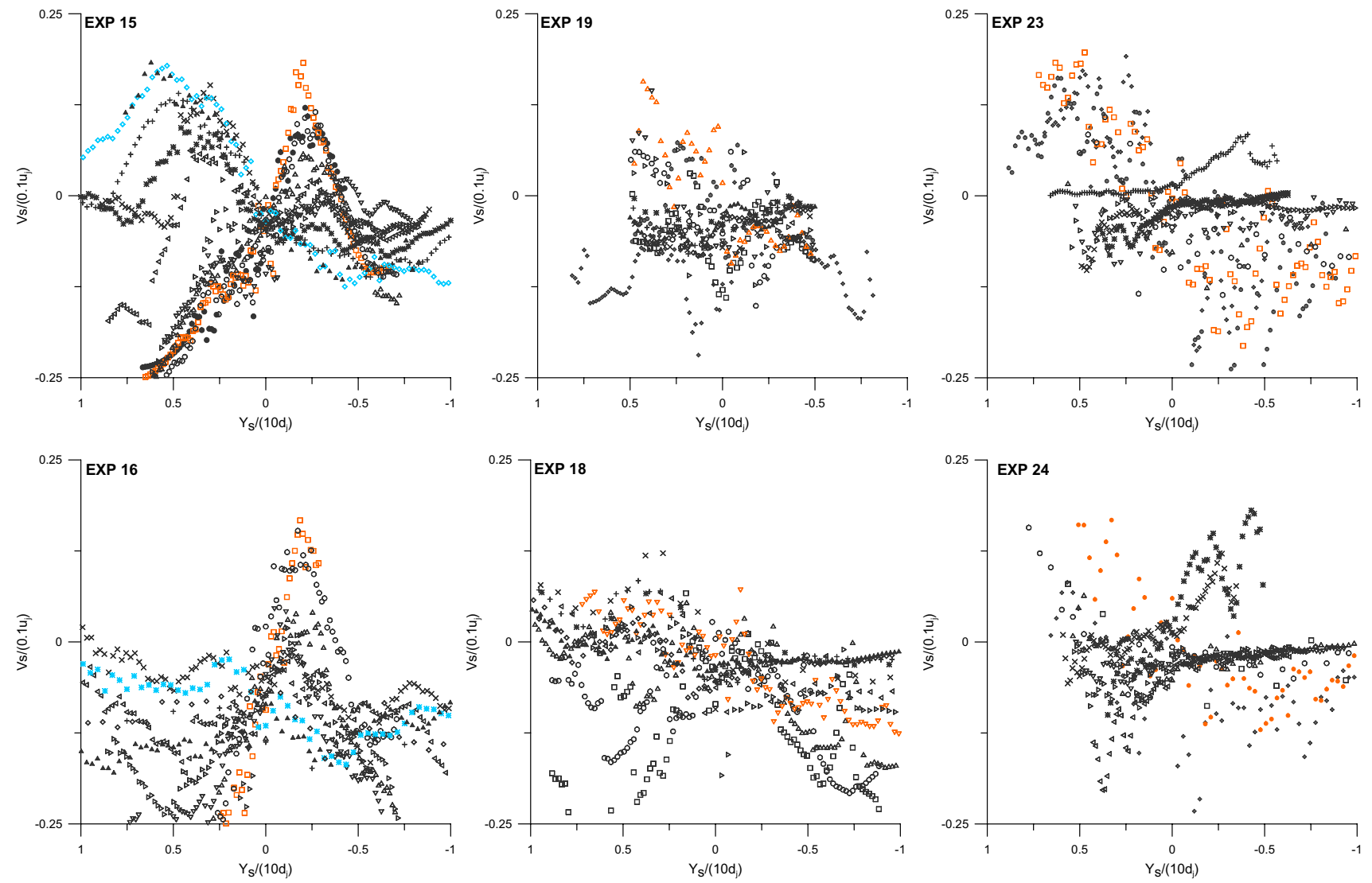

Fig. 6 Profiles along the examined sections of the dimensionless spanwise jet velocities. Top row $T=90 \mathrm{~s}$ and bottom row $T=60 \mathrm{~s}$. Same symbols as in Fig. 5. Colored plots in the online version of the paper 
Fig. 7 Trend of the jet width $B$ along the jet trajectory. Left $T=90 \mathrm{~s}$ and right $T=60 \mathrm{~s}$

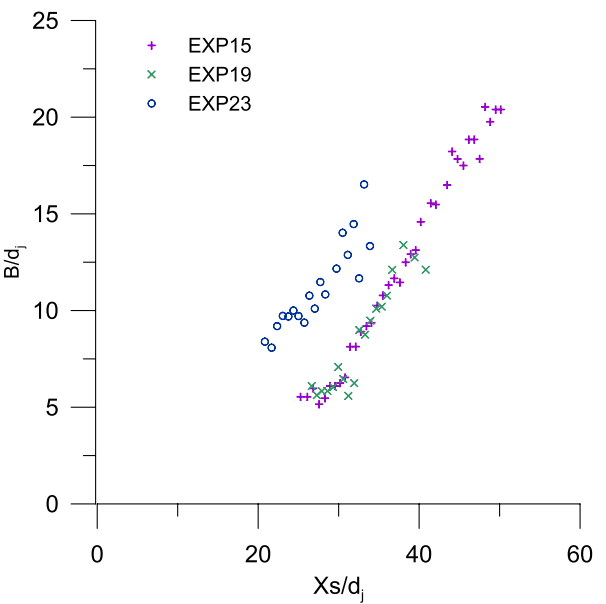

distances $X s / d_{j}$ in EXP16 and EXP18, while they are greater in EXP24 and are reached at lower distances from the origin. The slopes of these linear trends (i.e. the coefficients of proportionality between $B$ and $X s$ ) are 0.25 for high-density cases and 0.35 for both low-density obstruction array and unobstructed cases. Consequently, we can deduce that the jet dispersion (i) begins closer to the outlet for higher density array cases, (ii) is less rapid, over an equal distance along $X s$, for unobstructed and low density obstruction array cases.

Figure 8 displays the trend of $U s / u_{j}$ varying along the jet trajectory $X s / X s_{0}$. The exponential decreasing trend for $U s$ is most evident for EXP15 and EXP16, similarly to free jets, starting from the region where the jet is well defined (Fig. 4), i.e. $X s / X s_{0}>2$. Exponentially decreasing trends for $U s$ values along $X s / X s_{0}$ are also observed for all other cases, with generally high values of the correlation coefficient (Fig. 8). It is worth noting that while in the unobstructed cases a loss close to $50 \%$ of the initial value of $U s$ occurs along a range on the trajectory equal to $2 X s / X s_{0}$, in the obstructed cases it is much more rapid, occurring inside the canopy, within $0.5 X s / X s_{0}$. This is especially evident for EXP19, where the initial values of $U s$ are comparable with those of EXP15 and EXP16, but it is also valid for EXP23 and EXP24.

Since the vertical velocity component was not measured, we define a time-averaged turbulent kinetic energy in the horizontal plane as $K=\frac{1.33}{2} \overline{\left(u^{\prime 2}+v^{\prime 2}\right)}$, following Ting and Kirby (1996). The prime symbol indicates the turbulent fluctuations of the considered velocity component, and the overbar indicates time-averaging over the measurement period. As displayed in Fig. 9, the reduction of $U s$ is accompanied by the decay of $K$, with faster decay rates in presence of obstacles. For the unobstructed experiments, we note a clear exponential decreasing trend for $K$ along the jet trajectory. In the experiments with the higher obstruction array density, the decay is twice more rapid along the jet axis. For the same obstruction array density, along the jet path we observe higher values of $K$ at higher rotation rate, indicating higher turbulent activity. This implies that, in our experiments, rotation has an impact also on the turbulent velocities, in the region where the jet is well developed.

The transport of $K$ by the mean flow, that is by the streamwise and spanwise velocity ( $U s K$ and $V s K$, respectively), is
Fig. 8 Trend of the streamwise velocity along the jet trajectory, with fitting exponential curves

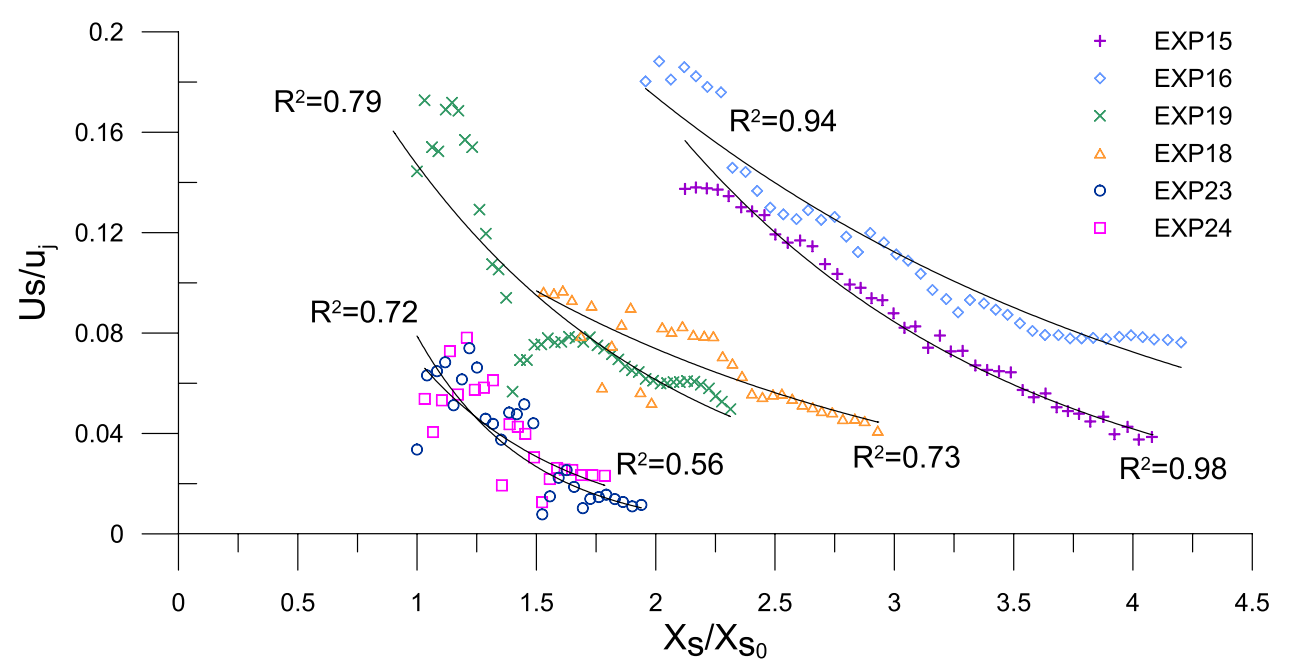


analyzed along the jet trajectory, for each examined section. Based on the lower order of magnitude of $V s$ with respect to $U s$, the spanwise transport is generally lower than the streamwise one, despite the transversal jet dispersion. In Fig. 10, the transport $U s K / u_{j}{ }^{3}$ is displayed for some selected sections along the trajectory, showing peak values in the jet core, close to the trajectory. Moreover, it reduces in presence of obstructions, with a faster decay rate for higher density arrays, confirming that in such cases the jet mixes faster with the ambient fluid.

All the obtained results show that neglecting the presence of porous obstructions in the environment where the jet is issued leads to overestimation of spreading of both the jet streamwise velocity and its turbulent kinetic energy.

\subsection{Jet trajectory and maximum penetration length of the jet}

We apply the dimensional analysis to better investigate the trends of jet trajectory deviations, in the simplified hypothesis of a not vertically nor horizontally confined jet (i.e. sufficiently deep water and large tank). Thus, the main variables governing the phenomenon are the kinematic momentum of the jet $M_{j}$, the rotation rate $\Omega$, the water density $\rho$ and molecular viscosity $\mu$, a characteristic horizontal length scale of the jet $L$, and the drag force per unit planar area exerted by the obstructions $\sigma$.

In this approach, in fact, the obstructions are accounted for by the drag force exerted in the $X$ and $Y$ directions, being the sum of the form and viscous drag components over the rod. As shown by Nepf (1999), various resistance laws for flow in porous media can be derived for such cases. Particularly, in open channel or in atmospheric vegetated flow, the quadratic form can be assumed to express the drag force per unit planar area:

$\sigma_{i}=\frac{1}{2} \rho C_{D}\left|u_{i}\right| u_{i}$ with $i=1,2$ and $u_{1}=u ; u_{2}=v$ in the current case.

In Eq. (1) the drag coefficient $C_{D}$ is shown, which depends on density and diameter of the obstacles (Nepf 1999). The unit planar area addressed in Eq. (1) is $a=n d$, also equal to $d / s^{2}$ in the case of a periodic square array, with $n$ being the density of the rods, i.e. the number of obstructions per unit planar area.

Consequently, we can express the studied phenomenon as a function $f(M, \Omega, \rho, \mu, L, \sigma)=0$. If we define the kinematic momentum of the jet $M_{j}$ as the product $u_{j} Q_{j}$, we can consider $M_{j}$, the water density $\rho$, and the length scale $L$ as three independent variables, thus arriving at equivalent function of the following non-dimensional numbers:

$f\left(\frac{M_{j}^{1 / 2}}{2 \Omega L^{2}}, \frac{\rho M_{j}^{1 / 2}}{\mu}, \frac{\sigma L^{2}}{\rho M_{j}}\right)=0$

The first no-dimensional number in Eq. (2) $\frac{M_{j}^{1 / 2}}{2 \Omega L^{2}}$ represents the measure of the inertial/Coriolis balance. When it becomes equal to unity, we derive a specific horizontal length scale of the jet $L_{0}=\frac{M_{j}^{1 / 4}}{(2 \Omega)^{1 / 2}}$, which can be considered as the threshold length corresponding to the balance of inertial and Coriolis force. This means that the value of $L_{0}$ constraints the dispersion of the jet in the rotating background when other actions are absent. For larger lengths, the rotation effect prevails over the inertial one. In our experiments, we compute $L_{0}=0.76 \mathrm{~m}$ and $L_{0}=0.62 \mathrm{~m}$, respectively for $T=90 \mathrm{~s}$ and $T=60 \mathrm{~s}$.

The second term in Eq. (2) can be rewritten as $\frac{\rho M_{j}^{1 / 2}}{\mu}=\frac{\rho L u_{j}}{\mu}$, thus being a jet-based Reynolds number, remaining constant in our study. The third number can be rewritten as $\frac{\sigma L^{2}}{\rho M_{j}}=\frac{\sigma}{\rho u_{j}^{2}}$, that is the jet drag coefficient $C_{D}$.

Therefore, rewriting the first non-dimensional number in terms of $L_{0}$, Eq. (2) can be rewritten as
Fig. 9 Trend of the turbulent kinetic energy along the jet trajectory, with fitting exponential curve for unobstructed cases

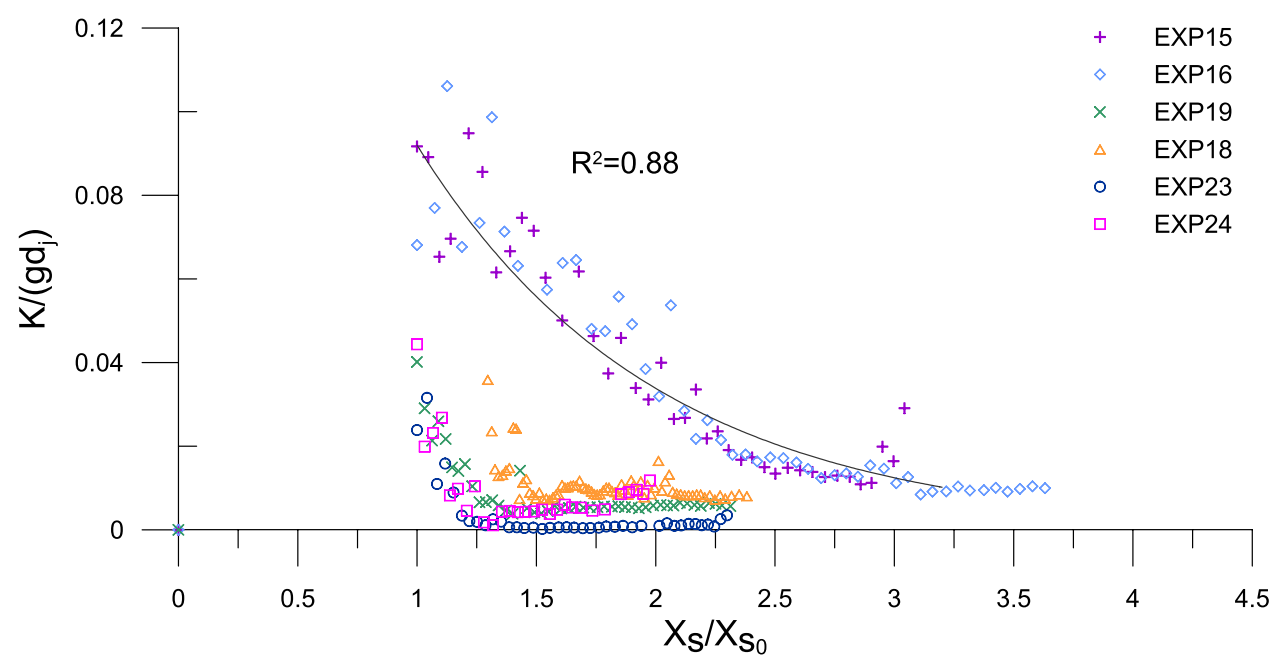



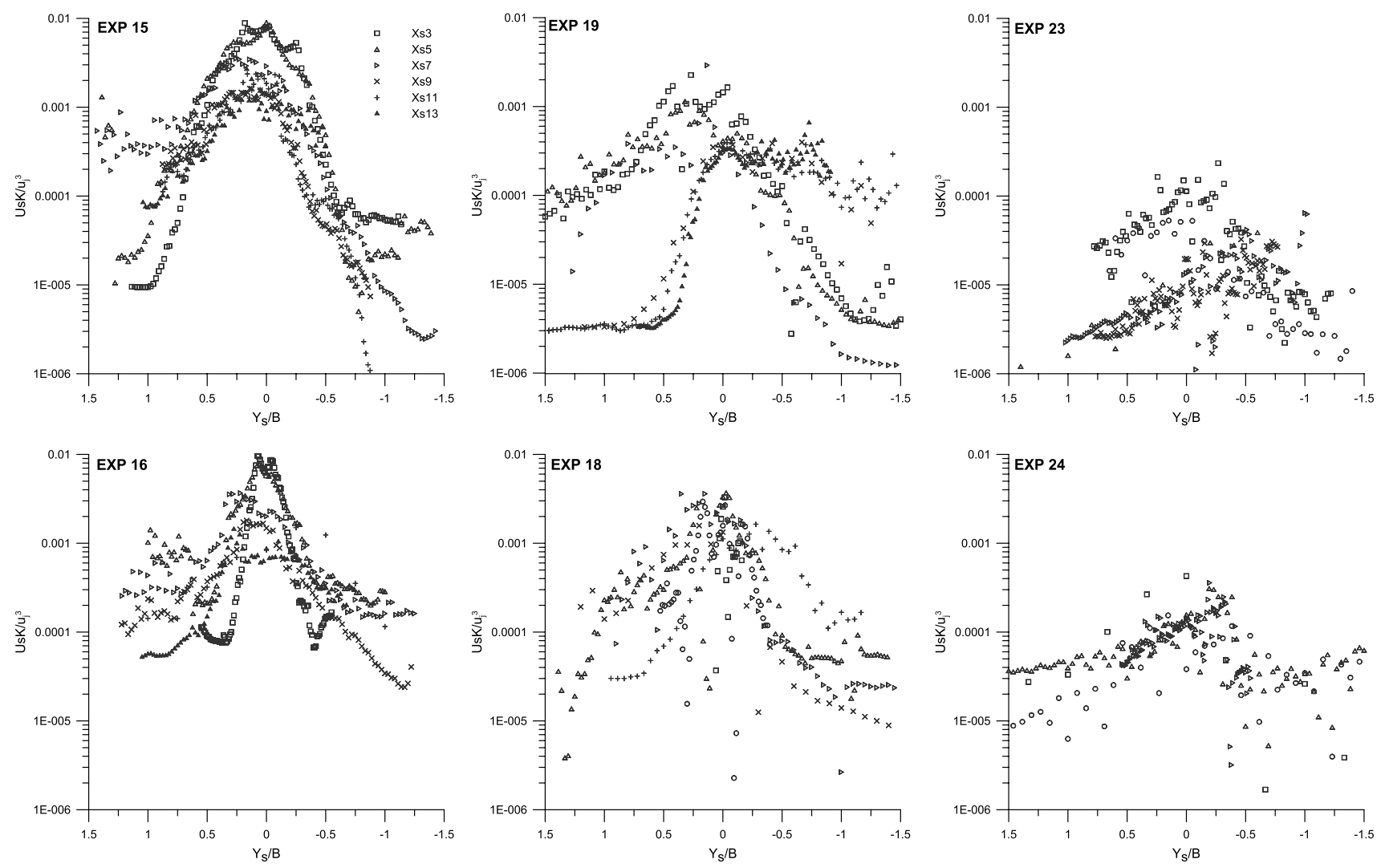

Fig. 10 Profiles along selected sections of the transport of jet turbulent kinetic energy by the mean flow. Top row $T=90 \mathrm{~s}$ and bottom row $T=60 \mathrm{~s}$

$f\left(\frac{L_{0}^{2}}{L^{2}}, C_{D}\right)=0$

or analogously as

$L=L_{0} \cdot f_{1}\left(C_{D}\right)$.

We take into consideration that when the obstructions are absent $C_{D}=0$ and thus Eq. (4) must reduce to $L=\beta L_{0}$, which is formally analogous to what was already found by Etling and Fernando (1994) in the case of unobstructed jets, with $\beta$ being a coefficient depending on the experimental conditions. Consequently, we now propose to assume for the function $f_{1}$ the exponential form $f_{1}=\beta e^{C_{D}}$, thus providing

$L=\beta L_{0} e^{C_{D}}$,

In our experiments, the characteristic horizontal scale $L$ in the rotating tank is taken to be the maximum jet penetration distance from its outlet, named $L_{C}$. Its coordinates in the fixed reference system are $(X c, Y c)$. Its values, for all the experiments, confirm that rotation effect prevails on the jet inertial one. Figure 11 displays all the experimental trajectories scaled by $L_{C}$, demonstrating their collapse on a single curve. In this figure, we also plot the experimental results of Sobey (1972) for an unobstructed momentum jet of $0.79 \mathrm{~cm}^{4} / \mathrm{s}^{2}$, issued in a tank of $5 \mathrm{~m}$ diameter and rotating with $\Omega=0.0751 \mathrm{rad} / \mathrm{s}$. Nevertheless, in Sobey's tests (1972) the value of $M_{j}^{1 / 2} /\left(2 \Omega L^{2}\right)$ is in the range similar to that of experiments, allowing a comparison of jet behavior. Our current results, compared with those of Sobey's (1972), confirm jets trajectories are homothetic.

To evaluate the coefficient $C_{D}$ we follow suggestion of Nepf (1999) for our values of $a d=0.01$ and $a d=0.04$, that correspond, respectively to our cases with sparse and dense obstacles array. We thus obtain $C_{D}=0.7$ for EXP 19 and EXP 23, and $C_{D}=0.4$ for EXP 18 and EXP 24. Figure 12 illustrates the comparison between the experimental $L c$ values and the theoretical ones obtained by the dimensional analysis with Eq. (5), revealing a high correlation $\left(R^{2}=0.9\right)$, supporting the validity of the proposed dimensional analysis approach. The calibration of the coefficient $\beta$ provides $\beta=5.55$ for the unobstructed cases and $\beta=2.25$ for the obstructed cases.

This result is of high significance, allowing estimation of the possible maximum jet penetration distance in both cases of absence or presence of obstacles, based only on known parameters of the jet (i.e. $\mathrm{Mj}$ ), of the rotating frame (i.e. $\Omega$ ), and of the obstruction array (diameter and density of the rods). Such 


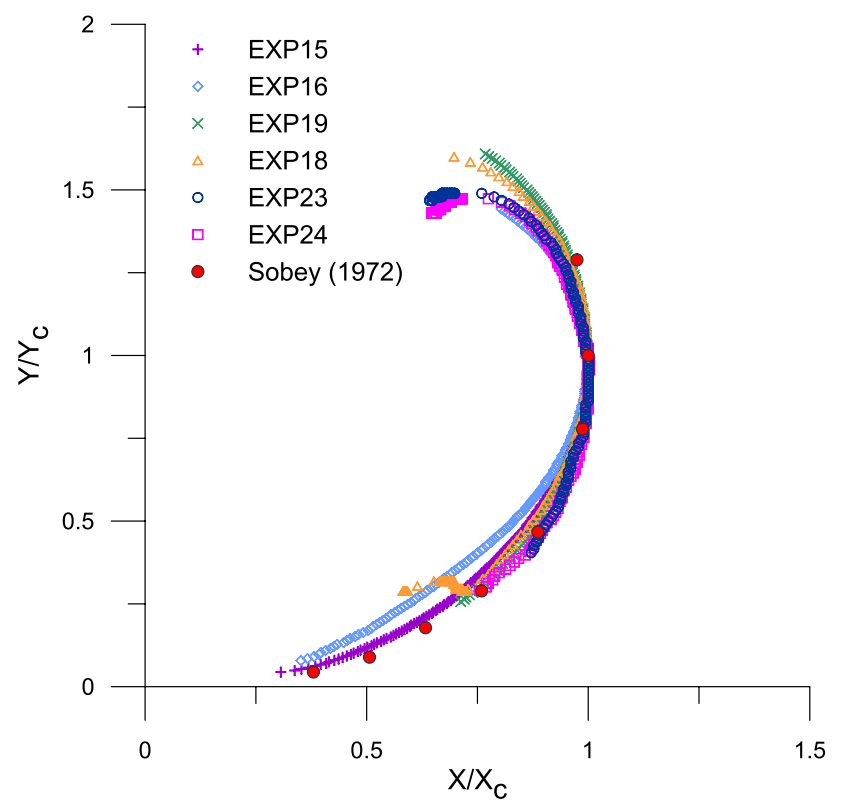

Fig. 11 Jet trajectories derived in all the investigated experiments, scaled by the characteristic length $L c(X c, Y c)$ observed in each experiment. Data by Sobey (1972) are superimposed

information may, for example, allow evaluation of the effective deltaic area influenced by a river issuing in a vegetated canopy, or evaluation of the actual distance at which cages or pens in a coastal farm could be affected by any polluting coastal discharge, thus rendering our findings important in planning and managing engineering activities in coastal areas.

\section{Conclusion}

The present study means to broaden a more thorough knowledge of momentum jets issued in obstructed environments, under the effect of Coriolis force. The experiments were carried out in the large-scale Coriolis platform at LEGI Grenoble (FR), where a large set of velocity data was obtained by means of a PIV system while varying the rotation rate of the tank and the density of the obstructions. The main results of this experimental analysis can be summed up as in the following.

The rotation evidently affects the jet, inducing its propagation trajectory curving; at the same time, the jet velocity reduces with increasing distance from the outlet. Presence of obstructions array determines a more rapid decrease of the velocity magnitude along the jet trajectory. The rate of velocity decrease is higher when the array density is higher; while for equal array density, it is higher at greater tank rotation rates, thus manifesting the effect of Coriolis on the jet inertia.

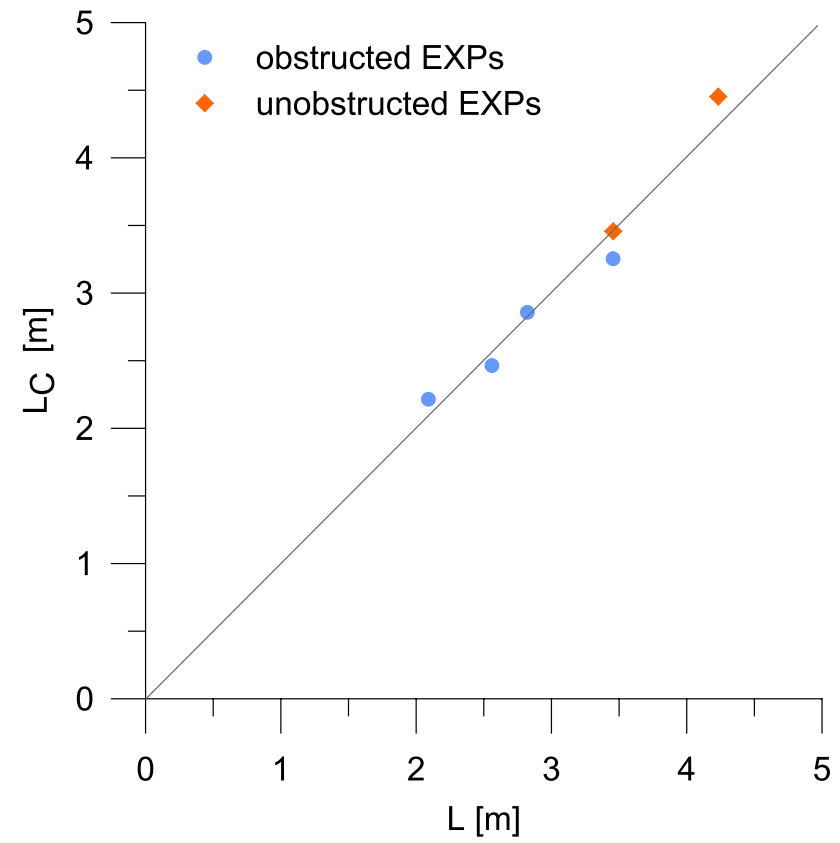

Fig. 12 Experimental characteristic length $L c$ plotted versus same length $L$ derived from Eq. (5) using the dimensional analysis

The profiles of the time-averaged streamwise and spanwise velocity along the examined cross-sections show that the former has higher rates of decrease along jets trajectories. For unobstructed cases, a Gaussian shape, typical for free jets, is observed in the profiles of the streamwise component. As well, the jet resembles the self-similarity behavior. The spanwise velocities are antisymmetric with respect to the jet axis and specifically in the obstructed experiments show a jet detrainment towards the ambient flow inside the array, confirming previous results of Mossa and De Serio (2016) derived in absence of rotation. The variability of the jet width along the trajectory path highlights that the jet dispersion begins closer to the outlet for higher density array cases; nevertheless, over equal distances, it is less rapid for unobstructed and low-density obstruction array cases.

The exponential decreasing trend of the streamwise velocity along the jet trajectory is evident for unobstructed cases, similarly to free jets. Its abatement rate is higher in the obstructed cases than in absence of obstructions. The turbulent kinetic energy was also observed to decay, at exponential rate in the unobstructed cases. Such decay is more rapid with higher obstruction array density. These results are relevant, as they show that neglecting the presence of porous obstructions in the environment where the jet is issued means overestimating the spreading of both jet streamwise velocity and jet turbulent kinetic energy. 
Finally, in all our experiments, a characteristic horizontal scale is observed, that is the maximum jet penetration distance from its outlet, which scales all the experimental trajectories, being them homothetic. The study proves that this scaling length is a function of jet momentum, rotation rate, and drag coefficient of the canopy. A simple expression is proposed and validated for such maximum length, thus being a useful tool in the planning and managing engineering activities of a coastal area.

Acknowledgments The experiments were carried out in the frame of the project H+-CNRS-06-JEVERB that received funding from the European Union's Horizon 2020 research and innovation program under grant agreement No. 654110, HYDRALAB+. All the authors are grateful to dr. Elvira Armenio and dr. Annalisa Di Leonardo for their cooperation during the execution of the experiments.

Author contributions FDS contributed to conceptualization. FDS, JS, MM contributed to methodology. GRP, RHG, DT, SV, TV, MEN, JS contributed to experiments. RHG, DL, FDS, MM contributed to data processing and analysis. FDS contributed to writing original draft. All the authors discussed and revise the paper.

Funding Open access funding provided by Politecnico di Bari within the CRUI-CARE Agreement.

Data availability The data that support the findings of this study are openly available in Zenodo repository at https://zenodo.org/record/ 4543130 .

\section{Declarations}

Conflict of interest The authors declare that they have no known competing financial interests or personal relationships that could have appeared to influence the work reported in this paper.

Open Access This article is licensed under a Creative Commons Attribution 4.0 International License, which permits use, sharing, adaptation, distribution and reproduction in any medium or format, as long as you give appropriate credit to the original author(s) and the source, provide a link to the Creative Commons licence, and indicate if changes were made. The images or other third party material in this article are included in the article's Creative Commons licence, unless indicated otherwise in a credit line to the material. If material is not included in the article's Creative Commons licence and your intended use is not permitted by statutory regulation or exceeds the permitted use, you will need to obtain permission directly from the copyright holder. To view a copy of this licence, visit http://creativecommons.org/licenses/by/4.0/.

\section{References}

Bailey BN, Stoll R (2016) The creation and evolution of coherent structures in plant canopy flows and their role in turbulent transport. J Fluid Mech 789:425-460. https://doi.org/10.1017/jfm.2015.749

Ben Meftah M, Mossa M (2016) A modified log-law of flow velocity distribution in partly obstructed open channels. Environ Fluid Mech 16(2):453-479. https://doi.org/10.1007/s10652-015-9439-7
Ben Meftah M, De Serio F, Malcangio D, Mossa M, Petrillo AF (2015) Experimental study of a vertical jet in a vegetated crossflow. J Environ Manag 164:19-31. https://doi.org/10.1016/j. jenvman.2015.08.035

Cossu R, Wells MG, Wåhlin AK (2010) Influence of the Coriolis force on the velocity structure of gravity currents in straight submarine channel systems. J Geophys Res 115:C11016

Davarpanah Jazi S, Wells MG, Peakall J, Dorrell RM, Thomas RE, Keevil GM, Darby SE, Sommeria J, Viboud S, Valran T (2020) Influence of Coriolis force upon bottom boundary layers in a large-scale gravity current experiment: Implications for evolution of sinuous deep-water channel systems. J Geophys Res Oceans 125(3):e2019JC015284

De Serio F, Ben Meftah M, Mossa M, Termini D (2018) Experimental investigation on dispersion mechanisms in rigid and flexible vegetated beds. Adv Water Resour 120:98-113. https://doi.org/ 10.1016/j.advwatres.2017.08.005

De Serio F, Armenio E, Badin G, Di Leonardo A, Hilel Goldshmid R, Liberzon D, Mossa M, Negretti ME, Pisaturo GR, Righetti M, Sommeria J, Termini D, Valran T, Vermeulen B, Viboud S (2019) Jet Interacting With Vegetation in a Rotating Basin. In: Proceedings of the HYDRALAB+ Joint User Meeting, Bucharest, May 2019

Etling D, Fernando HJS (1994) On the influence of background rotation on turbulent jets. In: Davies PA and Valente Neves MJ (eds) Recent Research Advances in the Fluid Mechanics of Turbulent Jets and Plumes, Nato Science Series E, vol 255, pp 401-411

Fernando HJS, Ching CY (1993) Effects of background rotation on turbulent line plumes. J Phys Oceanogr 23:2125-2129

Ghisalberti M, Nepf H (2005) Mass transfer in vegetated shear flows. Environ Fluid Mech 5(6):527-551. https://doi.org/10.1007/ s10652-005-0419-1

Godeferd FS, Moisy F (2015) Structure and dynamics of rotating turbulence: a review of recent experimental and numerical results. Appl Mech Rev 67:030802-030811. https://doi.org/10.1115/1. 4029006

Hussein JH, Capp SP, George WK (1994) Velocity measurements in a high-Reynolds-number, momentum-conserving, axisymmetric, turbulent jet. J Fluid Mech 258:31-75. https://doi.org/ 10.1017/S002211209400323X

Liberzon D, Fernando HJS (2014) Pressure distribution in confined jet flow. J Fluids Eng 136(3):031202. https://doi.org/10.1115/1. 4026438

Lin G, Atkinson JF (2000) A mechanism for offshore transport across the Gulf Stream. J Phys Oceanogr 30:225-232. https://doi.org/ 10.1175/1520-0485(2000)030\%3c0225:amfota\%3e2.0.co;2

Liu C, Shan Y, Nepf HM (2021) Impact of stem size on turbulence and sediment resuspension under unidirectional flow. Water Resour Res 57: E2020WR028620. https://doi.org/10.1029/ 2020WR028620.

Moisy F, Morize C, Rabaud M, Sommeria J (2011) Decay laws, anisotropy and cyclone-anticyclone asymmetry in decaying rotating turbulence. J Fluid Mech 666:5-35. https://doi.org/10. $1017 /$ S0022112010003733

Mossa M, De Serio F (2016) Rethinking the process of detrainment: jets in obstructed natural flows. Sci Rep 6(1):39103. https://doi. org/10.1038/srep39103

Mossa M, Ben Meftah M, De Serio F, Nepf HM (2017) How vegetation in flows modifies the turbulent mixing and spreading of jets. Sci Rep 7:6587. https://doi.org/10.1038/s41598-017-05881-1

Mossa M, Goldshmid R, Liberzon D, Negretti ME, Sommeria J, Termini D, De Serio F (2021) Quasi-geostrophic jet-like flow with obstructions. J Fluid Mech. https://doi.org/10.1017/jfm. 2021.501 
Nepf HM (1999) Drag, turbulence, and diffusion in flow through emergent vegetation. Water Resour Res 35:479-489. https://doi.org/10. 1029/1998WR900069

Nepf H (2012) Hydrodynamics of vegetated channels. J Hydraul Res 50(3):262-279. https://doi.org/10.1080/00221686.2012.696559

Nepf H, Mugnier C, Zavistoski R (1997) The effects of vegetation on longitudinal dispersion. Est Coast Shelf Sci 44:675-684. https:// doi.org/10.1006/ecss.1996.0169

New TH, Lim TT, Luo SC (2006) Effects of jet velocity profiles on a round jet in cross-flow. Exp Fluids 40:859-875. https://doi.org/ 10.1007/s00348-006-0124-y

Oldham CE, Sturman JJ (2001) The effect of emergent vegetation on convective flushing in shallow wetlands: scaling and experiments. Limnol Oceanogr 46(6):1486-1493. https://doi.org/10.4319/lo. 2001.46.6.1486

Poggi D, Krug C, Katul GG (2009) Hydraulic resistance of submerged rigid vegetation derived from first-order closure models. Water Resour Res 45:W10442. https://doi.org/10.1029/2008W R007373

Praud O, Sommeria J, Fincham AM (2006) Decaying grid turbulence in a rotating stratified fluid. J Fluid Mech 547:389-412. https:// doi.org/10.1017/S0022112005007068

Raupach MR, Thom AS (1981) Turbulence in and above plant canopies. Ann Rev Fluid Mech 13:97-129. https://doi.org/10.1146/ annurev.fl.13.010181.000525

Righetti M (2008) Flow analysis in a channel with flexible vegetation using double-averaging method. Acta Geophys 56(3):801-823. https://doi.org/10.2478/s11600-008-0032-z
Sami S, Carmody T, Rouse H (1967) Jet diffusion in the region of flow establishment. J Fluid Mech 27(2):231-252. https://doi.org/10. 1017/S0022112067000291

Smith SH, Mungal MG (1998) Mixing, structure and scaling of the jet in crossflow. J Fluid Mech 357:83-122. https://doi.org/10.1017/ S0022112097007891

Sobey RJ (1972) Flow Patterns in Lakes and Reservoirs, Ph.D. Dissertation, Imperial College of Science and Technology, University of London, London

Termini D (2015) Flexible vegetation behavior and effects on flow conveyance: experimental observations. Int J River Basin Manag 55(3):423-429. https://doi.org/10.1080/15715124.2015.1012519

Termini D (2018) River processes and Links between fluvial and coastal systems in a changing climate. Adv Water Resour 120:1-3. https://doi.org/10.1016/j.advwatres.2018.05.011

Thomas PJ, Linden PF (2007) Rotating gravity currents: small-scale and large-scale laboratory experiments and a geostrophic model. J Fluid Mech 578:35-65. https://doi.org/10.1017/S002211200 7004739

Ting FCK, Kirby JT (1996) Dynamics of surf-zone turbulence in a spilling breaker. Coast Eng 27:131-160. https://doi.org/10.1016/ 0378-3839(95)00037-2

Wang C (2018) ParaPIVlab: PIVlab in Parallel. doi: https://doi.org/10. 13140/RG.2.2.30705.79203. https://www.researchgate.net/publi cation/322550884_ParaPIV_PIVlab_in_Parallel.

Publisher's Note Springer Nature remains neutral with regard to jurisdictional claims in published maps and institutional affiliations. 